\title{
El enfoque de género en las noticias de salud
}

\author{
María Teresa Ruiz ${ }^{\mathrm{a}}$ Marta Martín ${ }^{\mathrm{b}}$ / Daniel La Parra ${ }^{\mathrm{b}}$ / Carmen Vives ${ }^{\mathrm{a}}$ / Manuel Albaladejo ${ }^{\mathrm{c}}$ \\ aDepartamento de Salud Pública. Universidad de Alicante. Alicante.

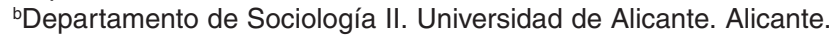 \\ 'Departamento de Filología Inglesa. Universidad de Alicante. Alicante. España.
}

Correspondencia: M. Teresa Ruiz Cantero. Departamento de Salud Pública. Universidad de Alicante. Apartado de correos 99. 03080 Alicante. España.

Correo electrónico: cantero@ua.es

Recibido: 30 septiembre de 2003. Aceptado: 16 de diciembre de 2003.

(The gender approach in health-related newspaper articles)

\section{Resumen}

Objetivos: Analizar con enfoque de género el contenido de las noticias sobre los problemas de salud que afectan a ambos sexos (cáncer, infarto y tabaco), o principalmente a mujeres (anorexia, malos tratos y aborto), publicadas en los medios de comunicación escritos durante los años noventa, y desarrollar algunas recomendaciones para la elaboración de noticias de salud con enfoque de género.

Material y métodos: Análisis del contenido de las noticias de los temas mencionados de El País, ABC y El Mundo (19911999). Fuentes de información: bases de datos informatizadas de los periódicos. Muestra de 1.358 noticias que contenían los términos seleccionados en la edición Nacional (malos tratos [57], anorexia [79], infarto [118], aborto [330], tabaco [350], cáncer [422]). Conceptos estudiados: «visibilidad», atribución de poder y paridad, y estudio con informadores claves.

Resultados: Un 38\% de las noticias que identificaban el sexo de los periodistas fueron firmadas por mujeres. Como actores de las noticias, los hombres (73\%) fueron más «visibles» que las mujeres (40\%). La mayor presencia de las mujeres fue como pacientes (14\%) y la de los hombres como políti$\cos (29 \%)$ y médicos (24\%).

Conclusiones: Pese a los esfuerzos realizados en los noventa, falta fortalecer el enfoque de género en las noticias sobre la salud.

Palabras clave: Medios de comunicación. Género. Paridad. Adquisición de poder. Sexismo. Infarto. Cáncer. Tabaco. Malos tratos. Anorexia. Aborto.

\begin{abstract}
Objectives: To use a gender approach to analyze the content of health-related articles affecting both sexes (cancer, heart attack and smoking) or mainly women (anorexia, domestic violence and abortion), published in newspapers in the 1990s. To provide recommendations for writing news with a gendersensitive approach.
\end{abstract}

Material and methods: Analysis of the above-mentioned topics in articles published in the national Spanish daily newspapers El País, ABC and El Mundo (1991-1999). Data sources: computerized databases of the newspapers. Sample of 1358 articles published in the national edition containing selected terms (battering [57], anorexia [79], heart attack [118], abortion [330], smoking [350], cancer [422]). Concepts studied: visibility, empowerment and equality. Interview with key informers.

Results: Thirty-eight percent of newspaper articles that identified the sex of journalists were signed by women. As the main protagonists of the feature, men $(73 \%)$ were more visible than women (40\%). Women mainly appeared as patients (14\%) whereas men mainly appeared as politicians $(29 \%)$ and physicians (24\%).

Conclusions: Despite the efforts made in the 1990s, the gender approach in health-related newspaper articles should be increased.

Key words: Media. Gender. Equality. Empowerment. Sexism. Myocardial Infarction. Cancer. Smoking. Battering. Anorexia. Abortion.

\section{Introducción}

E n la Cuarta Conferencia Mundial de Naciones Unidas sobre la Mujer celebrada en Beijing (China) ${ }^{1}$, España suscribía los Acuerdos de la mujer y los medios de comunicación, como uno de los retos a lograr antes del año 2000 para la promoción de la igualdad entre hombres y mujeres. Se plantearon 2 objetivos estratégicos: "Aumentar el acceso de las mu- jeres a la expresión de sus ideas y a la adopción de decisiones en los medios de difusión y por conducto de ellos». Es decir, incrementar su «visibilidad» y capacidad de actuación en los medios, y «fomentar una imagen equilibrada y no estereotipada de las mujeres en los medios de difusión", o impulsar un trato paritario y no sexista. Para ello, se instó a gobiernos, empresas de comunicación, y asociaciones profesionales a tomar medidas, como la creación de órganos de autorregu- 
lación y vigilancia de los medios de difusión, que elaboraran directrices profesionales y códigos de conducta, que incluyeran el enfoque de género en sus mecanismos de control y autocontrol, para velar por la «visibilidad» y la equidad de género.

La vida de los ciudadanos a título individual, y el buen funcionamiento de la sociedad en conjunto, está parcialmente subordinada al flujo de la información pública. En un entorno cada vez más global y segmentado, la comunicación colectiva es imprescindible, pues tiene la capacidad de señalar globalmente las amenazas para el sistema, contribuye a la conciencia pública de situaciones que pueden convertirse en problemas sociales, y difunde posibles soluciones².

Los periodistas exploran la sociedad para detectar excesos o anomalías ${ }^{3}$, y algunas son incluidas en sus agendas, para darlas a conocer a la opinión pública. De esta manera, los medios son los filtros más potentes entre el conocimiento público de los temas y la ignorancia ${ }^{3}$. Este tamiz no es imparcial. Los encuadres periodísticos de los temas no son neutros. Por ideología o como resultado de las limitaciones impuestas por la propia comunicación, los medios simplifican su labor recogiendo las partes más llamativa de los temas, e influyen con ellas en las definiciones subjetivas de los receptores, y en las decisiones públicas y políticas $^{3,4}$. Por tanto, las instituciones informativas tienen una tarea definitoria de la realidad. Y además, una vez presentado el tema, mediante los espacios informativos se proponen causas y se adjudican soluciones, de modo que las agendas mediáticas ejercen inevitablemente una tarea de liderazgo con importantes repercusiones en las soluciones que se proponen ${ }^{4}$.

Los medios son capaces de construir un problema social producto de los valores culturales y de las rutinas profesionales de los periodistas. Dando a conocer y definiendo hechos amenazantes en muchos lugares y a muchas personas simultáneamente, pueden transformar situaciones indeseables (con componentes puramente objetivos) en auténticos problemas sociales (con componentes, además, subjetivos). Pero no todas las disfunciones captan la atención de los medios y las convierten en problemas sociales. Es más, por amenazadora que resulte una situación, la atención pública que se le va a prestar a lo largo del tiempo es limitada ${ }^{5-7}$.

Los medios, quieran o no los periodistas y las empresas informativas, son una poderosa herramienta para la salud pública, puesto que las noticias sobre los temas de salud y enfermedad con frecuencia cumplen los criterios de noticiabilidad, y además son las fuentes de información de temas de salud más utilizadas por la población, y en ocasiones por los propios profesionales sanitarios e incluso la comunidad científica ${ }^{8}$.

Sin embargo, cada vez con mayor frecuencia se va manifestando una incomodidad con la realidad de las mujeres que reflejan los medios de comunicación, en la medida que es parcial, y con el tipo de periodismo que se practica, en la medida que a menudo es discriminatorio con las mujeres y fortalecedor de valores erróneos. Se cuestionan los hechos que son considerados noticia, la idea de mujer que se transmite, la escasez de mención a personas del sexo femenino, a qué se les vincula, cuál es la relación entre los sexos que se desprende de la lectura y las conclusiones a las que llevan las informaciones objeto de noticias ${ }^{9-14}$.

El objetivo de este trabajo ha sido analizar con enfoque de género («visibilidad» o aparición pública, «empoderamiento» y paridad) el contenido de las noticias de problemas de salud que afectan a ambos sexos (cáncer, infarto y tabaco), o principalmente a mujeres, ya sean clásicos o emergentes (anorexia, malos tratos y aborto), publicadas en los medios de comunicación escritos durante la década de los noventa. Los objetivos específicos han sido, en primer lugar, determinar la aparición pública de hombres y mujeres en las noticias, tanto en la firma (autores/as), como en el título y cuerpo de la noticia; en segundo lugar, identificar la contribución de las noticias al «empoderamiento» de hombres y mujeres, mediante las categorías profesionales o personales en que ambos sexos son presentados, incluida la imagen de culpabilización de las personas que aparecen en las noticias; en tercer lugar, examinar la paridad de las noticias, mediante la comparación de los roles -sujetos de acción, de opinión, o sujetos pacientescon los que hombres y mujeres en sus categorías profesionales o personales son presentados.

\section{Material y métodos}

En la primera fase del estudio se ha realizado un análisis del contenido de las noticias -de cáncer, infarto, anorexia, malos tratos, aborto y tabaco- publicadas durante la década de los noventa, en los 3 diarios nacionales de mayor tirada según la Oficina de Justificación de la Difusión: El País, ABC y El Mundo. En concreto, para recoger información de las noticias publicadas antes y después de la Conferencia de Beijing (1995), se realizó una selección no aleatoria de los años 1991, 1993, 1996 y 1999, excepto para El Mundo, en el que se estudiaron los años 1996 y 1999 debido a que las fuentes de información fueron las bases de datos informatizadas existentes en los propios periódicos.

De una población total de noticias $(n=2.480)$-cuyo titular o contenido comprendía uno o varios de los siguientes términos: cáncer, infarto, anorexia, malos tratos, aborto y tabaco-, se seleccionaron todas las noticias $(n=1.358)$ pertenecientes a la edición nacional: 57 de malos tratos, 81 de anorexia, 118 de infarto, 330 de aborto, 350 de tabaco y 422 de cáncer. Por medios, el número de noticias fue de 586 en El País, 342 en El Mundo y 316 en $A B C$. 
Para la codificación de las noticias se elaboró un protocolo con 158 variables y un manual para codificadores. Para la codificación se entrenó a un hombre (filólogo) y a una mujer (socióloga). La concordancia en la clasificación de las variables estudiadas en el análisis de 100 noticias fue superior al $80 \%$.

Partiendo de la definición de sesgo de género ${ }^{14}$, se acordó que éste existía en las noticias de salud, siempre y cuando uno de los 2 sexos reciba un tratamiento informativo perjudicial, en especial, cuando se compara con el recibido por el otro sexo. Para ello, los conceptos estudiados han sido: «visibilidad», «empoderamiento" y paridad.

Para medir la «visibilidad» o su falta, se trabajó con las variables no excluyentes referentes a la presencia de hombres y mujeres en la firma (autores/as) y propiamente en la noticia.

Uno de los indicadores de «empoderamiento» o nivel de control de mujeres y hombres sobre su propia salud es observar quién tiene el protagonismo en las noticias de salud: si en la noticia el protagonismo -en cuanto a presencia- corresponde a políticos o profesionales, ésta contribuirá escasamente a una visión de los pacientes como dueños de su propia salud. O si, por el contrario, los pacientes son el centro de la noticia, ésta contribuirá a dar una imagen en la que el proceso de enfermedad o salud corresponde precisamente a quien la padece o disfruta. Se cuantificó y comparó la presencia de hombres y mujeres en función de variables no excluyentes relativas a las categorías profesionales -médicos/as, académicos/as, escritores/as, famosos/as de los ámbitos del espectáculo, cultura y deportes, y políticos/as-y las categorías personales - pacientes o víctimas de malos tratos y familiares de pacientes-. Por otra parte, se cuantificó la culpabilización de la víctima en la noticia.

La paridad es un concepto próximo al de «visibilidad» que añade a la igualdad numérica que la mide la igualdad en las aportaciones que hombres y mujeres hacen a la noticia. Por tanto, se ha entendido como falta de paridad cuando, dentro de una misma categoría profesional o personal de los sujetos de la noticia, se producen desequilibrios de las acciones desempeñadas por cada sexo, ya sea en su papel de sujeto de la acción que daba lugar a la noticia (hacer) de opinión (decir) o sujetos pasivos del hecho que daba lugar a la noticia (padecer). En función de las variables no excluyentes relativas a las categorías profesionales y personales, se cuantificó y comparó la presencia de hombres y mujeres como sujetos de acción, opinión y padecimiento de las noticias. Este método también se reprodujo para el examen de la paridad según las noticias fueran de cáncer, infarto, anorexia, malos tratos, aborto y tabaco.

Las técnicas estadísticas para el análisis del contenido fueron básicamente la descripción de frecuen- cias y porcentajes y el desarrollo de tablas de contingencia. Para la realización de este análisis se ha utilizado el programa estadístico SPSS10.

En la segunda fase de estudio, y con el fin de producir recomendaciones para la elaboración de noticias de salud con enfoque de género, un total de 14 informadores clave, profesionales (incluido el equipo de investigación) expertos/as en o de los medios de comunicación y del ámbito académico relacionado con la comunicación, filología, salud y feminismos, participaron en una sesión de trabajo de un día de duración, durante el cual se analizaron los conceptos de «visibilidad", paridad y sexismo en el lenguaje en las noticias de anorexia, aborto, cáncer, infarto, tabaco y malos tratos a la mujer.

Previamente a la reunión de trabajo se envió a cada experto/a un informe en el que se sintetizaba la información resultante del análisis del contenido de las noticias. Junto al informe se incluyó un cuestionario centrado en cómo eliminar los sesgos de género relacionados con la «visibilidad», la paridad y el sexismo en el lenguaje en las noticias de los temas mencionados anteriormente, para que lo respondieran y enviaran antes de la fecha fijada para la reunión de trabajo. Sus respuestas fueron presentadas junto con los principales resultados del análisis del contenido para dar comienzo a la reunión. Una vez finalizada, se extrajeron las conclusiones que dieron lugar a 12 recomendaciones para la elaboración de noticias de salud con enfoque de género.

\section{Resultados}

En la figura 1 se puede observar la evolución de las noticias de los diferentes temas de salud estudiados. Sobre todo el cáncer y el tabaco son los temas que han acaparado el interés mediático, con una tendencia creciente durante los años. Todo lo contrario que lo que sucede con el aborto.

La «visibilidad» de las mujeres, como autoras de las noticias, sigue estando por debajo de la de los hombres aunque, como se puede observar en la figura 2 , se está incrementando el número de informaciones en las que la periodista firma su trabajo. De las 742 noticias en las que se pudo identificar el sexo de los/las periodistas, un $60 \%$ fueron firmados por hombres, y un $38 \%$, por mujeres. Sólo un 1,6\% incluyó en su rúbrica a ambos. En los temas tradicionales fue predominante la firma de los hombres: aborto (un $77 \%$ de autores frente al $20 \%$ de autoras), infarto (el 65 frente al $31 \%$ ), cáncer (el 54 frente al $45 \%$ ) y tabaco (el 61 frente al $37 \%$ ), mientras que en los temas emergentes (anorexia y malos tratos) aparecieron más rúbricas de mujeres que de hombres (un 59\% de autoras frente al 31\% 


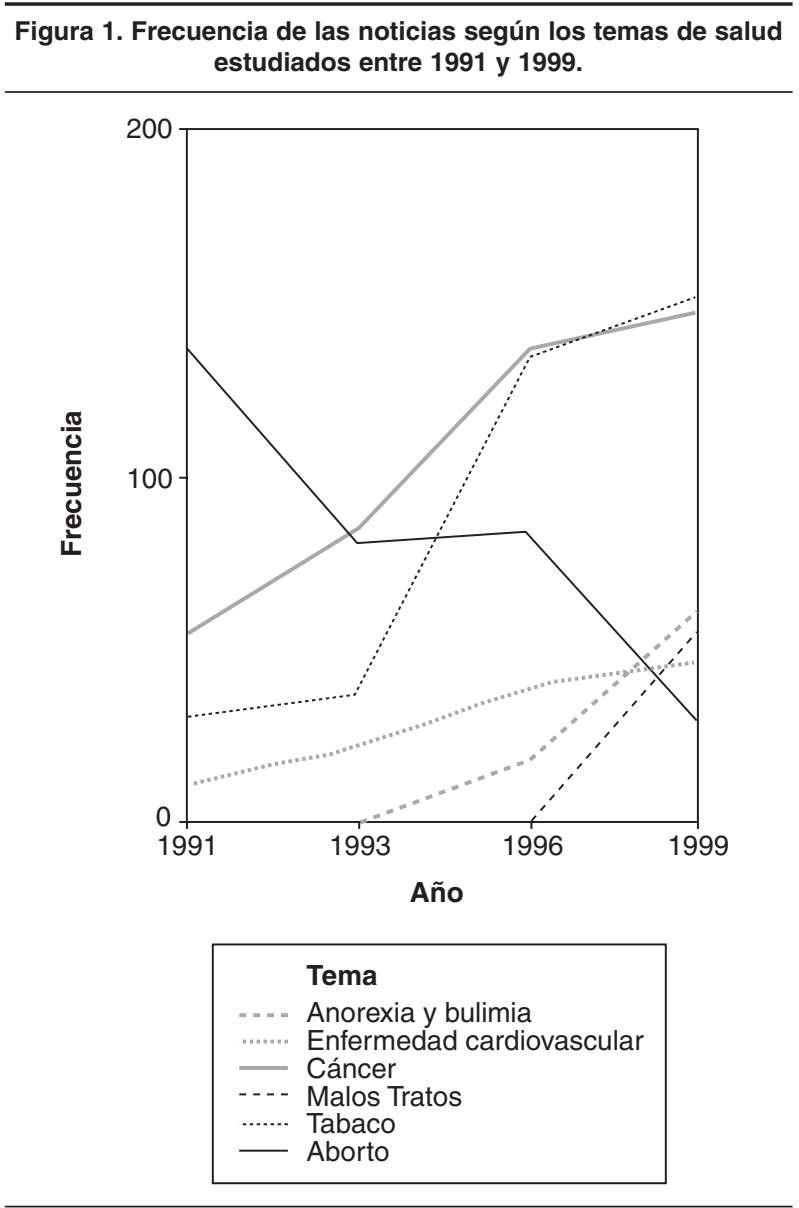

de autores, y un $71 \%$ de autoras frente al $25 \%$ autores, respectivamente).

Ya en las noticias, los hombres fueron más «visibles» que las mujeres: en un $71,7 \%$ aparecieron hombres y en un $38,3 \%$ aparecieron mujeres. Aunque la diferencia se hace mayor al analizar las noticias según aparezcan hombres o mujeres en exclusiva, pues en un $41,6 \%$ aparecen solamente hombres y en un $8,2 \%$ aparecieron únicamente mujeres. Por temas (tabla 1), sólo en los malos tratos el umbral de presencia de las mujeres superó al de los hombres, casi quintuplicándolo (el 24,6 frente al 5,3\%). No sucedió lo mismo con la anorexia, donde la presencia de mujeres y hombres fue si-

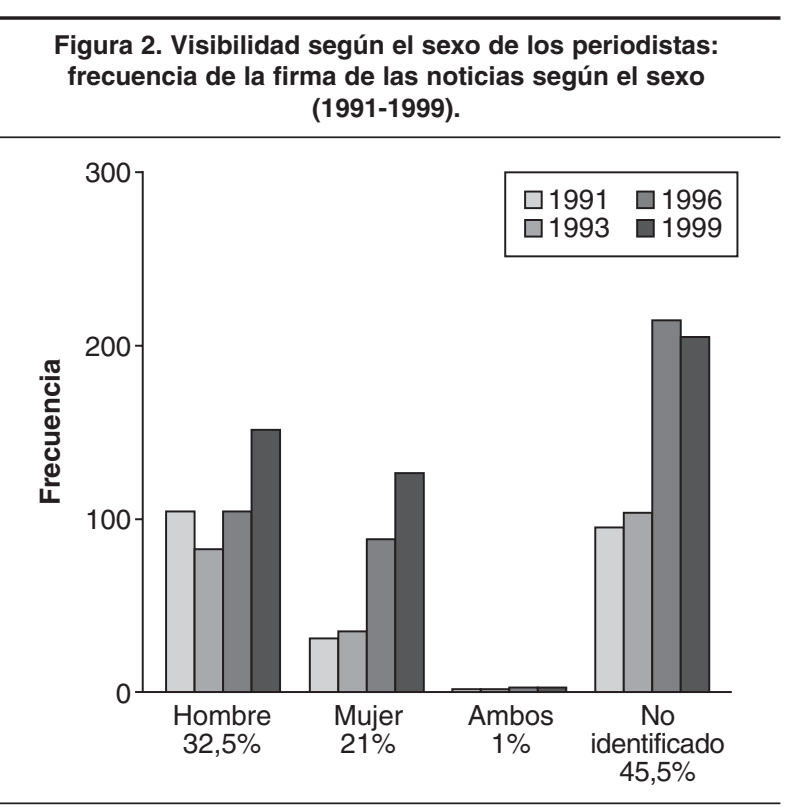

milar (el 19 frente al 22,8\%). Por el contrario, las mujeres son relativamente «invisibles» en las noticias sobre infarto, cáncer, tabaco y aborto. Así, hay una escasa presencia de las mujeres en las noticias de infarto $(2,5 \%)$ en comparación con la mucha mayor presencia de hombres $(50,8 \%)$, cáncer (el 8,3\% en las mujeres frente al $41,9 \%$ en los hombres) y tabaco (el 6,6 frente al 52,9\%). Llama la atención la frecuente presencia de hombres $(36,7 \%)$ en las noticias de aborto respecto a la de mujeres $(6,4 \%)$. De forma complementaria, se quiere destacar que en el análisis diacrónico de las noticias de cada uno de los temas hay una tendencia constante de mayor «visibilidad» masculina, con excepción del aumento relevante de mujeres en el tiempo en las noticias sobre cáncer y tabaco.

Si bien la tabla 2 permite múltiples lecturas relacionadas con la adquisición de poder, sobresale la menor cabida en el espacio mediático de las mujeres y los pacientes, pues aparecen desplazadas frente a los médicos y políticos hombres. Pero lo más destacable es que el rol con mayor representación por parte de la población femenina en los medios es el de enferma.

Tabla 1. «Visibilidad» de mujeres y hombres (\%) en las noticias sobre diversos problemas de salud publicadas en EI País, ABC y EI Mundo (1991-1999)

\begin{tabular}{|c|c|c|c|c|c|c|c|}
\hline & Anorexia & Infarto & Cáncer & Malos tratos & Tabaco & Aborto & $\%$ Total \\
\hline Ni hombres ni mujeres & 20,3 & 13,6 & 19,9 & 15,8 & 27,4 & 15,8 & 20,1 \\
\hline Hombres & 22,8 & 50,8 & 41,9 & 5,3 & 52,9 & 36,7 & 41,6 \\
\hline Mujeres & 19,0 & 2,5 & 8,3 & 24,6 & 6,6 & 6,4 & 8,2 \\
\hline Hombres y mujeres & 38,0 & 33,1 & 29,9 & 54,4 & 13,1 & 41,2 & 30,1 \\
\hline Frecuencia & 81 & 118 & 422 & 57 & 350 & 330 & 1.358 \\
\hline
\end{tabular}


Tabla 2. Principal presencia según la categoría profesional y personal y sexo ${ }^{\mathrm{a}}$, en las noticias de diversos problemas de salud ${ }^{\mathrm{b}}$ publicadas en EI País, ABC y EI Mundo (1991-1999) $(n=1.358)$

\begin{tabular}{|c|c|c|c|c|c|}
\hline \multicolumn{3}{|c|}{$\begin{array}{l}\text { Rango, frecuencias y porcentajes } \\
\text { de noticias en las que hay mujeres }\end{array}$} & \multicolumn{3}{|c|}{$\begin{array}{l}\text { Escala, frecuencia y porcentajes } \\
\text { de noticias en las que hay hombres }\end{array}$} \\
\hline & Frecuencia & $(\%)$ & & Frecuencia & $(\%)$ \\
\hline & & & 1. Políticos & 470 & $(34,6)$ \\
\hline & & & 2. Médicos & 339 & (25) \\
\hline 3. Pacientes & 230 & $(16,9)$ & & & \\
\hline \multirow[t]{4}{*}{ 4. Políticas } & 178 & $(13,1)$ & & & \\
\hline & & & 5. Científicos & 158 & $(11,6)$ \\
\hline & & & 6. Familiares & 97 & $(7,1)$ \\
\hline & & & 7. Pacientes & 91 & $(6,7)$ \\
\hline 8. Familiares & 77 & $(5,7)$ & 8. Famosos & 78 & $(5,7)$ \\
\hline 9. Médicas & 71 & $(5,2)$ & & & \\
\hline 10. Famosas & 50 & $(3,7)$ & & & \\
\hline & & & 11. Escritores & 37 & $(2,7)$ \\
\hline 12. Científicas & 32 & $(2,4)$ & & & \\
\hline 13. Escritoras & 5 & $(0,4)$ & & & \\
\hline
\end{tabular}

avariables no excluyentes. ${ }^{D N}$ Noticias sobre cáncer, infarto, tabaco, malos tratos, anorexia y aborto

Por otra parte, también destaca que quienes padecen anorexia sean las personas a quienes más se culpabiliza (un $34,6 \%$ de las noticias de anorexia culpabilizaban a quienes la padecían). En segundo lugar, están las noticias sobre el aborto, pues el $16,4 \%$ de ellas culpabilizan a la embarazada o al médico que provocó el aborto. Siguen las noticias sobre el tabaco (un 10,9\% de las noticias culpabilizaban a los/las fumadores/as), las noticias sobre los malos tratos (un $7 \%$ de las noticias culpabilizan a la víctima) y, por último, el 1,7\% de las noticias sobre el cáncer también culpabilizan a quienes lo padecen. No se menciona este tema en las noticias de infarto.

En general, se han detectado problemas de paridad en las noticias estudiadas, pues no hay igualdad en las aportaciones de hombres y mujeres dentro de la misma categoría profesional (tabla 3). Son las acciones y opiniones de los políticos, los médicos y los científicos que no de sus compañeras de profesión, las que se reflejan con mayor frecuencia en las noticias. Como complemento específico a esta información, cabe mencionar que en un $79 \%$ de las noticias sobre el cáncer, los hombres desempeñaron un papel activo (haciendo y diciendo), mientras que las mujeres desempeñaron este papel en el $43,4 \%$ de las informaciones. En el caso de las noticias sobre tabaco, también los hombres lideraron la iniciativa (en el $90,6 \%$ de las informaciones en las que hubo hombres, éstos hicieron o dijeron), más que las mujeres (en un $82,5 \%$ de las noticias en las que aparecen mujeres, éstas hacen o dicen). Finalmente, en el $70,9 \%$ de las noticias sobre aborto, los hombres desempeñaron un papel activo diciendo o haciendo, mientras que este porcentaje fue del $38,7 \%$ en las mujeres.

Los resultados de la reunión de expertos/as quedan reflejados de forma sintetizada en las recomendaciones del anexo 1.

Tabla 3. Paridad en las noticias de diversos problemas de salud a publicadas en EI País, $A B C$ y EI Mundo. Principal presencia en rango, frecuencias y porcentajes de mujeres y hombres como sujeto de acción, opinión, o padecimiento en la noticia según su categoría profesional y personal ${ }^{\mathrm{b}}(\mathrm{n}=1.358)$

\begin{tabular}{|c|c|c|c|c|c|c|c|c|c|c|c|}
\hline \multicolumn{4}{|c|}{ Sujetos de la acción origen de la noticia } & \multicolumn{4}{|c|}{ Sujetos que opinan en la noticia } & \multicolumn{4}{|c|}{ Sujetos pacientes de la acción origen de la noticia } \\
\hline Mujeres & $\%$ & Hombres & $\%$ & Mujeres & $\%$ & Hombres & $\%$ & Mujeres & $\%$ & Hombres & $\%$ \\
\hline & & 1. Político & 21 & & & 1. Político & 22 & 1. Paciente & 16,1 & & \\
\hline & & 2. Médico & 14,9 & & & 2. Médico & 15,8 & & & 2. Médico & 6,8 \\
\hline & & 3. Científico & 7,9 & & & 3. Científico & 9,41 & & & 3. Paciente & 6,6 \\
\hline 4. Política & 7,1 & & & 4. Política & 9,1 & & & & & 4. Político & 4,3 \\
\hline \multirow[t]{3}{*}{ 5. Paciente } & 4,7 & & & 5. Médica & 3,2 & & & & & 5. Famoso & 2,7 \\
\hline & & 6. Familiar & 3,9 & & & 6. Famoso & 2,5 & & & 6. Familiar & 2,3 \\
\hline & & 7. Famoso & 3,6 & 7. Paciente & 1,8 & & & 7. Famosa & 1,5 & & \\
\hline 8. Familiar & 3,4 & & & & & 8. Familiar & 1,72 & 8. Médica & 1,2 & & \\
\hline 9. Médica & 2,6 & & & $\begin{array}{l}\text { 9. Familiar/ } \\
\text { Científica }\end{array}$ & 1,5 & & & 9. Familiar & 0,7 & & \\
\hline 10. Famosa & 1,8 & 10. Escritor & 1,8 & 10. Famosa & 1,3 & & & 10. Política & 0,5 & & \\
\hline \multirow{2}{*}{ 11. Científica } & 1,5 & & & & & 10. Escritor & 1,1 & & & 11. Científico & 0,4 \\
\hline & & 12. Paciente & 1,3 & & & 11. Paciente & 0,9 & 12. Escritora & 0,1 & 12. Escritor & 0,1 \\
\hline 13. Escritora & 0,2 & & & 12. Escritora & 0,1 & & & 13. Científica & 0 & & \\
\hline
\end{tabular}

aNoticias sobre cáncer, infarto, tabaco, malos tratos, anorexia y aborto. Variables no excluyentes. 
Anexo 1. Recomendaciones para la elaboración de noticias de salud con enfoque de género

1. Equiparar el tratamiento dado a los hombres y las mujeres en las noticias, tanto si se les sitúa en la esfera doméstica como en el espacio público. Reflejar la diversidad de roles que las mujeres desempeñan en relación con la salud en el ámbito privado y en el público.

2. Preguntarse rutinariamente sobre la presencia de mujeres en las noticias, y si no aparecen, manifestarlo de forma explícita. No olvidar que las mujeres son la mitad de la población y el 75\% (3 de cada 4) de quienes proporcionan cuidados «informales» de salud.

3. Diversificar las fuentes de información al tratar las noticias de salud-enfermedad para ofrecer un enfoque lo más multidimensional posible. No olvidar que las personas afectadas y su entorno son expertas en la resolución de sus problemas de salud y en procurarse calidad de vida; por tanto, es recomendable reconocer su autoridad e incluir en la noticia sus opiniones.

Desplazar la mirada e identificar nuevas fuentes en entornos diferentes (familia, sociedad civil, asociaciones de pacientes, de familiares de pacientes) a las instituciones oficiales.

4. Dar énfasis a la dimensión humana en el tratamiento de los problemas de salud. En la descripción de la noticia es conveniente incluir, además de términos médicos o técnicos, la dimensión emocional de los protagonistas de la noticia, así como su impacto social. Lo personal no tiene que ser menos riguroso; es conveniente encontrar el valor añadido en el relato de quienes tienen un problema de salud.

5. Para divulgar conocimiento e incorporarlo al discurso social, es necesario hacer comprensibles los problemas de salud, sin prescindir del uso de términos técnicos propios y específicos, pero adjuntando su debida explicación. Utilizar despieces gráficos con guías y con preguntas concretas que se pueden realizar a los especialistas próximos a la audiencia. También, incluir en las noticias referencias a fuentes directas, contrastando previamente su operatividad.

6. Construir una imagen positiva del proceso de cualquier enfermedad sin clichés androcéntricos. Tener en cuenta que la audiencia no es neutra y realiza inferencias personales a partir de la información obtenida, sea estadística o a partir de un caso concreto.

7. En la selección de temas y la redacción de noticias, evitar fomentar falsas expectativas y demandas innecesarias, evitando dar información que cree ansiedad, miedo y frustración (fomenta el sensacionalismo). Aplicar la prudencia en situaciones de crisis.

8. Sacar a la luz pública los problemas de salud derivados de estereotipos de género, e interpretar la información con perspectiva de género. La violencia ejercida contra las mujeres es un problema generado por una concepción masculina de la violencia, de las que ellas y ellos salen perjudicados.

9. Considerar la existencia y la forma que adoptan el sexismo de quien escribe y el sexismo de la audiencia. Revisar los textos comprobando si el lenguaje respeta la «visibilidad» de hombres y mujeres. Frecuentemente, se oculta el sexo de quienes protagonizan las noticias (investigadores, enfermeras, pacientes...). Algunas acciones que evitan la ambigüedad son: a) utilizar la dualidad de género (médico/médica, enfermero/ enfermera...); b) emplear los plurales masculinos y femeninos para reflejar lo más fielmente posible la composición del grupo de referencia; c) feminización de las profesiones; d) servirse del contexto para especificar la verdadera composición del grupo; e) recurrir a expresiones gramaticales que carezcan de género -adjetivos (sus), pronombres (quién/quienes)...-, y f) centrarse en la acción o profesión más que en los protagonistas o profesionales, y en los genéricos colectivos («equipo») más que en la función de sus participantes.

10. Describir a las personas con problemas de salud y a su realidad con palabras y expresiones que las representen con dignidad, y dejar de tratar las desviaciones de la norma como anomalías. Supone: a) describir a la persona, no denominarla por la enfermedad para no reducir el todo a una parte; $b$ ) referirse a la enfermedad sólo cuando sea relevante, y c) evitar las exageraciones y la provocación de pena, culpabilidad, estigmatización, resignación y menosprecio.

11. La fórmula más correcta para denominar a las personas en una noticia es por el nombre y apellidos, y si procede por cargo y profesión. Revisar las denominaciones de las personas que aparecen en la noticia, no mencionando a quien no tenga relevancia para el mensaje, y limitando las referencias de parentesco a los casos en que se intentara asegurar su anonimato.

12. En los temas de salud, respetar muy especialmente los derechos de la persona (honor, intimidad y propia imagen), garantizando el anonimato en los casos en que proceda (p. ej., aborto, malos tratos...).

\section{Discusión}

Los resultados inducen a concluir que, durante los años noventa, las acciones relacionadas con la comunicación en temas de salud han sido uno de los puntos probablemente menos desarrollados por los Planes de Igualdad. Pese a los esfuerzos de asociaciones de mujeres de los medios de comunicación y de académicas/os, falta fortalecer el enfoque de género en los mecanismos de autorregulación de la propia profesión 9-12,14,16.

La principal limitación del estudio -de carácter descriptivo pero con vocación constructiva- es que se centra en el análisis de la información producida por la prensa y falta el análisis del consumo de la audiencia y del impacto de estas noticias en ella. Además, en este sentido, la elección como objeto de estudio de El País, ABC y El Mundo por su capacidad de liderazgo o de marcar agenda y por ser preferentemente leídos por determinadas clases o grupos de elite, limita las conclusiones en cuanto a la imagen de las mujeres en los medios en los problemas de salud elegidos, y probablemente sería diferente si se hubieran analizado estas noticias en televisión, radio u otros soportes mediáticos destinados a mujeres. Por último, se ha realizado un análisis del contenido explícito, y queda pendiente analizar el discurso que los medios estudiados tienen sobre estos problemas de salud y que depende de su ideología.

Aunque, después de Beijing, casi se han duplicado las noticias firmadas por mujeres periodistas, la «invisibilidad» de las mujeres en los medios de comunicación comienza por ellas mismas. A similares conclusiones llega una reciente publicación realizada a partir del Informe Quiralt ${ }^{17}$. Este aumento puede re- 
lacionarse con el incremento de mujeres en las redacciones, o porque 2 de los temas seleccionados (anorexia y malos tratos), que ya eran tratados por mujeres con mayor frecuencia, han saltado a la arena pública en los últimos años.

La «visibilidad» de las mujeres es necesaria en toda la agenda mediática, pero especialmente en las noticias de salud. Su falta de «visibilidad» y la de sus intereses puede tener importantes consecuencias. Cuando ciertos problemas, que afectan sobre todo a las mujeres, no aparecen suficientemente reflejados en los medios, se contribuye a la falta de interés por resolverlos (como ha sido el caso de la violencia de género durante mucho tiempo). Cuando determinados tipos de noticias excluyen sistemáticamente la alusión a las mujeres, se favorece la percepción pública de que ciertas enfermedades se asocian a hombres (como sucede con las enfermedades cardiovasculares). Cuando los medios dejan de cubrir la esfera privada -y con ello la ocupación de un determinado sector de la población-, infravaloran actividades, que no son «visibles» pero que resultan fundamentales en el manejo y control de las enfermedades (como ocurre con los cuidados de salud familiares). Cuando los medios no reflejan en sus agendas los cambios producidos en las vidas de las mujeres, en cuanto a su mayor participación en el sector laboral remunerado (incluido el político, sanitario y científico o cualquier otra tarea de prestigio social), están contribuyendo a que las mujeres no se desarrollen en esos ámbitos ${ }^{7,18,19}$.

Pero los daños para una parte de la población no sólo se producen porque no aparezcan mujeres en los medios. El sesgo de género en las noticias de salud se produce, también, cuando las aportaciones de las mujeres en el campo de la salud o en otras esferas de la vida social no son valoradas, o la participación de ambos sexos en los procesos de toma de decisión no quede reflejada de forma equilibrada en los medios de comunicación. El tratamiento informativo desfavorable o perjudicial a las mujeres resta poder y, junto con su falta de paridad, contribuye a restarles legitimidad a la hora de ejercer autoridad, esto es, en la toma de decisiones ${ }^{19,20}$.

Se expone a continuación una serie de conclusiones específicas de las noticias de cada uno de los problemas de salud estudiados:

\section{Enfermedades crónicas: cáncer e infarto}

En las noticias sobre cáncer e infarto, se podría estar perpetuando una realidad sujeta a otros condicionantes extraperiodísticos, como los intereses económicos -en donde la medicina es un campo abonado para ellos-, las creencias científicas y populares, algunas de las cuales son erróneas; la réplica de sesgos, como el de género, que se producen dentro del sector sanitario.

Así, pese a la elevada mortalidad, las noticias sobre infarto no son un tema importante en la agenda mediática (en los años estudiados, son 3,5 veces menos causa de noticia respecto a las originadas por el cáncer). Precisamente, la hiperbólica atención al cáncer de mama de los medios puede ser uno más de los riesgos para la salud de las mujeres, en la medida que dándolo a conocer con mucha más frecuencia que otros problemas de salud más prevalentes en ellas -caso de las enfermedades vasculares- puede contribuir a que tanto las mujeres como los profesionales sanitarios y los políticos minusvaloren auténticas amenazas para las mismas.

La introducción de la perspectiva de género en las noticias sobre estos temas, además de ser una estrategia para facilitar cambios en las voces de las fuentes y las perspectivas de las noticias, es una herramienta clave más para garantizar que la información sea exacta, en la medida que ayuda a precisarlas.

\section{Violencia de género}

Durante años, las noticias de violencia en los hogares han sido ubicadas en la sección de sucesos. Sólo recientemente parece existir conciencia de que dicha violencia reproduce una pauta de desigualdad de género, pues la mayor parte de la violencia la ejercen hombres contra mujeres. Al adoptar esta visión, las noticias que aparecían de forma aislada han adquirido un sentido de conjunto, pasando a ser denominadas como noticias sobre malos tratos y violencia doméstica o de género. Así, los acontecimientos aislados, carentes de explicación, han pasado a ser encuadrados dentro de un esquema explicativo común: la violencia masculina dentro del hogar. Lo que ha impulsado a que desde otras esferas sociales se desarrollen propuestas para la solución a este «nuevo» problema. El caso de las noticias de violencia contra las mujeres ilustra que existen ciertas realidades sociales que permanecen ocultas o poco «visibles», y también que pueden llegar a ser «visibles».

Tabaco

Los medios de comunicación están realizando una campaña activa contra el tabaco, que tiene la particularidad de mantenerse como contenido de actualidad durante años. De los temas de salud analizados, sólo el tabaco ha tenido una amplia y constante cobertura durante la década. La presencia de mujeres en las noticias sobre el tabaco está aumentando, pero aún son, junto con las de cáncer e infarto, en las que hay menor presencia de mujeres. 
Es complejo identificar el impacto en la salud de esta campaña, en especial, en la de las mujeres, pues se necesita conocer la audiencia, los modos en que ésta se apropia de los contenidos informativos y hacer una descripción completa de las características de los mensajes. A partir de este trabajo difícilmente se puede ir más allá del análisis del mensaje. Así, cabe destacar que las noticias sobre factores de riesgo -tabaco en este caso- son especialmente proclives a la culpabilización de la víctima, y afectan en ocasiones a colectivos como el de las mujeres embarazadas.

\section{Anorexia}

La cobertura mediática es una fuerza importante para el inicio de un ciclo de interés público y político, y para transformar lo que era un problema raro en una preocupación pública. En un artículo paradigmático, Downs propuso que la historia de los problemas sociales es la historia de su ciclo de atención ${ }^{5}$. Igualmente, se podría afirmar que la historia de los ciclos de atención de los problemas públicos es la historia de su ciclo de noticiabilidad. Éste fue, para el caso de la anorexia, como sigue:

Fase preproblema. Antes de 1999, las noticias sobre la anorexia eran ocasionales. Los periodistas suelen detectar la mayor parte de los problemas aquí, cuando sólo son conocidos por sectores concretos de población (personas afectadas y expertas). En 1996 aumentan las noticias, enfocándolas como suceso anecdótico, lejano y glamuroso que afectaba a jóvenes modelos, o como problema médico en las secciones de ciencia y salud. Sólo esporádicamente se explicita la responsabilidad de sectores concretos, como el de la moda o publicidad.

Fase de descubrimiento alarmante del problema. En 1999, al indicar el riesgo, los medios de comunicación ponen en marcha el ciclo de interés público. Acontecimientos dramáticos de modelos y deportistas produjeron un alud puntual de noticias que visibilizaron la anorexia en los medios. El discurso cambia radicalmente y se presenta como un verdadero problema social con dimensiones políticas. Aquí es cuando la comunidad -poder político incluido- hace el descubrimiento alarmante del problema. Desde este momento, el tema traspasa la intimidad de las afectadas a la arena pública.

Fase de toma de conciencia de los costes de las soluciones. Pese a la complejidad etiológica y de la realidad de los trastornos de la alimentación, durante el debate político realizado en el Senado, los medios formularon el problema de la anorexia en términos de juicio público, donde presentaron al bando acusador (fundamentalmente afectadas/os y los políticos/as) y al de los acusados (sector de la moda, deporte y los propios medios de comunicación). Y aparecían noticias ocasionales con soluciones rápidas para paliar el problema: «El Ministerio convoca a los expertos para luchar contra la enfermedad» (El Mundo, 17-3-1999) o «Guerra a la talla 36» $(A B C, 16-3-1999)$.

Fase de decrecimiento gradual del interés. Aunque al comienzo del debate los colectivos directamente señalados (sobre todo la moda) se defendían y eximían de responsabilidades, al final las asumieron públicamente. En noviembre de 1999 se publicó el documento final sobre los trastornos de la alimentación elaborado por la Comisión del Senado (referido en casi todos los periódicos). En diciembre se observa un decrecimiento gradual de las noticias de anorexia.

Fase posproblema. Situación de baja atención sobre la anorexia o de recurrencias espasmódicas de interés. Los medios, que ya tienen en sus agendas otros problemas, contribuyen a que la población enfoque su atención hacia un nuevo tema. Frecuentemente, si en este período las instituciones o grupos interesados programan el tratamiento del tema, los medios pueden realizar una función de recuerdo, reiniciando una y otra vez el ciclo de noticiabilidad.

\section{Interrupción voluntaria del embarazo (IVE)}

Ningún otro tópico de salud de la mujer es tan ejemplarizante de la influencia de los grupos de poder en los discursos de los medios como el del aborto, por su cobertura y los ámbitos de influencia reflejados en las noticias. Los medios seleccionan los temas, centran su atención sobre determinadas fuentes y presentan a las audiencias a determinados personajes que creen más significativos y que responden a su ideología, quedando desplazadas las opiniones y valoraciones de otras fuentes tan o más protagonistas de la realidad de las IVE.

Existe abundante bibliografía sobre las razones por las que un tema aparece y desaparece de los medios. En el caso de la IVE parece bastante obvio que los medios reflejan, como foro de discusión que son y desde sus diferentes ideologías, el debate político producido en las dos últimas décadas del siglo xx. Por tanto, con la casi desaparición del debate público, a finales de los noventa, el silencio de la prensa ha sido la característica principal. Las noticias se redujeron a la tercera parte durante la última mitad de la década, y a la quinta parte durante toda ella. Además, las noticias sobre el aborto en la adolescencia han brillado por su ausencia en toda la década.

Pese a ser un problema que afecta sobre todo a las mujeres, los medios han escogido como interlocutores válidos en las noticias de aborto mayoritariamente a los hombres. Los autores de estas noticias son también en su mayoría periodistas hombres. 
Desde sus diferentes ideologías, los medios reflejan una realidad de confrontación social: "Lo que tenemos aquí es una guerra, y no vamos a detener nuestra lucha hasta que ganemos", dice el reverendo Keith Tucci (ABC, 6-4-1993). «Dios perdona siempre, el hombre, a veces, y parcialmente; pero la naturaleza, que no es persona, toma venganza», comenta el cardenal López Trujillo (ABC, 30-11-1991). Por otra parte, el papel de las mujeres cambia en las noticias sobre el aborto, pues al interrumpir voluntariamente el embarazo son reflejadas como culpables, en lugar del estereotipo clásico de pacientes con el que aparecían en las noticias de los otros temas analizados: "Cada vez son más las mujeres que prefieren privar al ser que han concebido, en este silencioso y lento autoexterminio que tiene sus raíces tanto en la penuria de todo tipo como en la degradación moral que han provocado siete décadas de régimen comunista» $(A B C, 17-7-1991)$. En este caso, había una doble culpa, por abortar y por socialistas, pues la noticia procedía de la ex Unión Soviética.

Es bastante frecuente que en las noticias sobre IVE no se respete el derecho a la intimidad pues se identifican los nombres y apellidos y ubicación de las mujeres que abortan y los de los médicos que realizan las intervenciones.

Por último, el espacio mediático se caracteriza por la ausencia de paridad en cuanto a los papeles desempeñados por los políticos de ambos sexos. De manera que hay poco espacio y escasas voces defendiendo argumentos en defensa de los derechos sexuales y reproductivos de la mujer, que es un argumento del feminismo socialista. Por tanto, podríamos estar percibiendo en las noticias sobre IVE el clásico debate entre los objetivos del socialismo y los feminismos.

\section{Conclusiones}

Los medios de comunicación y la libertad de prensa, instrumentos y garantía de los sistemas democráticos, también son uno de los principales instrumentos para aumentar el grado de democracia en las decisiones que afectan a la salud. En este sentido es preciso seguir incrementando el enfoque de género en las noticias de los problemas de salud estudiados. Así, más allá de mostrar el estereotipo de mujeres pacientes, aumentando la «visibilidad» y la voz de las mujeres con otros roles, los medios podrían contribuir a la defensa de sus derechos, al incremento de la pluralidad y diversidad de las fuentes de información y al aumento de la conciencia en la opinión pública, los partidos políticos y el resto de las instituciones sociales sobre la existencia de problemas de salud. Por último, aunque igualmente importante, los medios deberían canalizar la acción para resolver cuestiones legislativas, económicas, culturales o ideológicas que afectan a la salud de la población, y más en concreto la de las mujeres.

El posicionamiento de los medios observado, fundamentalmente en el modo en que muestran y tratan los temas de salud -tanto de forma positiva como negativa, o disminuyendo su presencia-, enseña a la audiencia pautas de valoración y procesos de afrontamiento de estos problemas de salud, aunque también culpabiliza a parte del público.

Hay numerosos ejemplos de prácticas de atribución de poder (democratizadoras sí se prefiere), y también de lo contrario. Las mujeres y los colectivos menos representados son los que más tienen que ganar o perder en este proceso.

\section{Bibliografía}

1. Cuarta Conferencia Mundial sobre la Mujer. Sección J. Los acuerdos de la mujer y los medios de comunicación en Beijing; 1995 [consultado 15/12/2003]. Disponible en: http://www.un.org/spanish/conferences/Beijing/mujer 2021.htm

2. Defleur M, Ball-Rockeach S. Theories of Mass Communication. New York: Logman; 1989.

3. McCombs M, Einsiedel E, Weaver D. Contemporary public opinion. Issues and the news. New Jersey: Lawrence Erlbaum Associates; 1991.

4. Gurevitch M, Levy M. Conceptualization and measurements of audience agenda. Mass Communication Review Yearbook. California: Sage; 1981.

5. Protess D, McCombs. Agenda-setting: readings on media public opinion, and policymaking. New Jersey: Lawrence Erlbaum Associates; 1991.

6. Rubington E, Weingberg MS. The study of social problems. Five perspectives. NewYork: Oxford University Press; 1977.
7. Lorber J. Gender and the social construction of illness. California: Sage; 1997.

8. Atkin C, Wallack L. Mass communication and public health. Complexities and conflicts. California: Sage; 1990.

9. Balletbó A. La mujer en los medios de comunicación social. Barcelona: Instituto de Ciencias de la Educación, Universidad Autónoma de Barcelona; 1982.

10. Fagoaga C, Fagoaga PM. Umbral de presencia de las mujeres en la prensa española. Madrid: Instituto de la Mujer, Ministerio de Cultura; 1987.

11. Fagoaga $C$. El género en los medios de comunicación. En: García de León MA, García Cortázar M, Ortega F, editores. Sociología de las mujeres españolas. Madrid: Editorial Complutense; 1996.

12. Radl R. Los medios de comunicación de masas y sus imágenes femeninas. En: García de León MA, García Cortázar $M$, Ortega F, editores. Sociología de las mujeres españolas. Madrid: Editorial Complutense; 1996.

13. Womenaction 2000. Contribución alternativa sobre mujer y medios de comunicación [consultado 15/12/2003]. Disponible en: http://www.womenaction.org/csw44/altrepspa.htm 
14. VI Encuentro de Contrainformación Mesa de Mujeres [consultado 15/12/2003]. Disponible en: http://www.unapalabraotra.org/femnet/contrainformacion.htm

15. Lenhart SH. Gender discrimination: a health and career development problem for women physicians. J Am Med Women Assoc 1993;48:155-9.

16. Associació de Dones periodistes de Catalunya. El sexe de la notícia. Reflexions sobre el gènere de la informació i recomanacions d'estil. Barcelona: Diputación de Barcelona; 1999.
17. Revuelta G, Alonso I, Tomas S, Guerrero M, Rohlfs I. Género y salud en la prensa diaria. Biomedia [consultado 15/12/2003] Disponible en: http://www.biomeds.net/biomedia/d02041103.htm

18. Van Dijk T. Racismo y análisis crítico de los medios. BarceIona: Paidós; 1987.

19. Durán M. Los costes invisibles de la enfermedad. Madrid: Fundación BBVA; 1999.

20. Chapman S, Lupton D. The fight for public health. Principles and practice of media advocacy. London: BMJ Publishing Group; 1994. 